Original Article (short paper)

\title{
Effects of linear and undulating periodization of strength training in the acceleration of skater children
}

\author{
Diego A. R. Jaimes ${ }^{1,4}$, Dennis Contreras ${ }^{3} \oplus$, Alejandra M. F. Jimenez ${ }^{1}$,

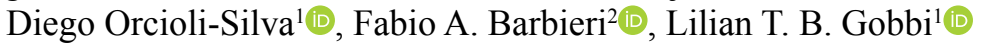 \\ ${ }^{1}$ Universidade Estadual Paulista, UNESP, Gait and Posture Studies Laboratory, Institute of Biosciences, Rio \\ Claro, SP, Brazil; ${ }^{2}$ Universidade Estadual Paulista, UNESP, Movement Research Laboratory, Faculty of \\ Sciences, Bauru, SP, Brazil; ${ }^{3}$ Universidad de Pamplona, Physical Education, Recreation and Sports Department, \\ Pamplona, Colombia; ${ }^{4}$ Universidad de Los Llanos, Physical Education Department, Villavicencio, Colombia
}

\begin{abstract}
Aim: This study aimed to compare the effects of linear periodization (LP) and undulating periodization (UP) of strength training on acceleration in skater children. Methods: Twenty-nine girls $(9.67 \pm 1.29$ years-old, $34.47 \pm 8.06 \mathrm{~kg}$, $1.39 \pm 0.13 \mathrm{~m}$ ) were distributed into two groups: linear periodization group (LPG, $\mathrm{n}=14)$ and undulating periodization group (UPG, $\mathrm{n}=15$ ). Six levels of progressive training were designed based on activities such as multi-jumps, plyometrics, sled towing, and facilitated exercises. The training lasted 16 weeks, with a 1-h session on three nonconsecutive days per week. The acceleration was recorded by digital videography following the major trochanter of the femur, at four moments and under two conditions: static (SS) and dynamic start (DS) in a rectilinear path. Statistical significance was set at 5\%. Results: ANOVA indicated group*moment interaction in SS and DS for velocity $\left(\mathrm{F}_{3,81}=7.883 ; \mathrm{p}<0.001 ; \mathrm{p} \eta^{2}=0.226 ; \mathrm{F}_{3,81}=2.36 ; \mathrm{p}=0.078 ; \mathrm{p}^{2}=0.08\right.$-trend, respectively $)$ and acceleration $\left(\mathrm{F}_{3,81}=3.96\right.$; $\mathrm{p}=0.011 ; \mathrm{p} \eta^{2}=0.128 ; \mathrm{F}_{3,81}=2.92 ; \mathrm{p}=0.039 ; \mathrm{p}^{2}=0.098$, respectively). Both groups increased velocity in $\mathrm{SS}$ and DS $\left(U P G / S S: 1^{\text {st }}=4.07,2^{\text {nd }}=9.75,3^{\text {rd }}=8.91,4^{\text {th }}=9.25 \mathrm{~m} / \mathrm{s} ;\right.$ LPG $/$ SS: $1^{\text {st }}=4.27,2^{\text {nd }}=7.13,3^{\text {rd }}=7.61,4^{\text {th }}=7.99 \mathrm{~m} / \mathrm{s} ; \quad U P G / D S:$

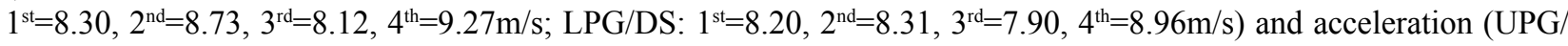
SS: $1^{\mathrm{st}=}=2.00,2^{\text {nd }}=8.69,3^{\mathrm{rd}}=4.71,4^{\mathrm{th}}=5.02 \mathrm{~m} / \mathrm{s}^{2} ;$ LPG $/ \mathrm{SS}: 1^{\mathrm{st}}=2.37,2^{\text {nd }}=3.39,3^{\mathrm{rd}}=3.68,4^{\mathrm{th}}=4.12 \mathrm{~m} / \mathrm{s}^{2} ; U P G / D S: 1^{\mathrm{st}}=1.78$, $\left.2^{\text {nd }}=1.97,3^{\text {rd }}=1.65,4^{\text {th }}=2.46 \mathrm{~m} / \mathrm{s}^{2} ; L P G / D S: 1^{\text {st }}=1.67,2^{\text {nd }}=1.70,3^{\text {rd }}=1.48,4^{\text {th }}=1.93 \mathrm{~m} / \mathrm{s}^{2}\right)$. Conclusion: Both strength training periodization protocols were effective in developing acceleration and velocity in girl skaters over 16 weeks; however, UP was more efficient than LP for improving acceleration.
\end{abstract}

Keywords: strength training periodization; inline speed skating; performance; load intensity; girls.

\section{Introduction}

The participation of children in muscle strength training programs has shown positive effects on sports performance ${ }^{1-3}$. This training provides adaptations in the child's body, such as increases in muscular strength levels and bone mineral density, improving sports performance ${ }^{4,5}$. This type of training improves power output, race speed, and change of direction, as well as overall motor performance ${ }^{1,3,6}$.

Strength training provides benefits in acceleration, which requires a great amount of force to "beat" the body's inertia. Good acceleration capability allows rapid changes in speed and direction of athletes and affords advantages in the performance of many sports, especially speed skating where the variables speed and acceleration are determinants of success during competitions?.

Different methods are used to improve muscle strength for the purpose of developing acceleration in skating, of which the two most common are the plyometric method ${ }^{8}$ and the method with external resistors, such as sled towing, elastic belt, climb skating. However, without proper periodization, the benefits of strength training may not be achieved. Previous studies have indicated that undulating periodization (UP) models generate significant improvements compared to linear models in athletes?. The most appropriate method of periodization for children, especially in speed skating, is relatively unknown $n^{5,10}$. Linear periodization (LP) seems to present benefits for muscular strength performance of children ${ }^{11,12}$, as it allows both gradual progressions and control of the training evolution ${ }^{5}$, without the necessity to combine the components of periodization ripples ${ }^{13}$. On the other hand, there is evidence that UP improves muscular strength in young athletes ${ }^{5,14,15}$. The advantages of UP over LP are the load waves allowed by UP, which facilitate the super compensation process and avoid possible plateaus of sports performance, a common fact in training programs with $\mathrm{LP}^{16}$. In addition, UP allows the combination of exercise methods through modulation of training load intensity, which is a relevant point of UP as it generates positive interconnections of the load, which may be more beneficial for performance stimulation in childhood.

In strength training periodization for children, the importance of the application of early age progressive intervention programs is highlighted, since it is during this period that high levels of neuronal plasticity occur ${ }^{8}$, favoring gains in muscle strength ${ }^{15}$. 
Thus, the aim of this study was to compare the effects of LP and UP of strength training on acceleration in skater children. The hypothesis of the study was that the UP model would provide greater gains in acceleration in skater children.

\section{Methods}

\section{Experimental Approach to the Problem}

A 2-group repeated-measures experimental design was applied to examine the effect of UP versus LP on acceleration performance in skater children for a static and dynamic start. Novice skater children were randomly distributed into a UP group (UPG) and LP group (LPG). The strength training was based on six levels of increasing intensity, applied over a 16-week period. The LPG developed all the training levels in ascending order of intensity, in this way, starting with the first low-intensity level and finishing at the sixth level, maximum intensity. The UPG developed the training program mixing training levels, maximum 2 per mesocycle. The regular skating training was the same for both groups. All the skaters were familiarized with the assessment protocol before starting the intervention. One week was established for each evaluation moment, applied at end of every mesocycle (4 microcycles per mesocycle). The acceleration assessment was performed 48 hours after the final training session, preceded by a 15-minute warm-up and followed by a 10-minute cooldown. All evaluations were conducted on the same day and time for every skater (17:00-19:00hrs) and the children used the same set of wheels that they used at the first evaluation moment.

\section{Subjects}

Twenty-nine girls, age $=9.67 \pm 1.29$ years, body mass $=$ $34.47 \pm 8.06 \mathrm{~kg}$, height $=1.39 \pm 0.13 \mathrm{~m}$, training experience in skating $=1.5 \pm 0.2$ years, from the infant category in speed skating of the Speed Cats team of the city of Villavicencio, participated in the study. The participants were randomly assigned to two groups according to the type of periodization: $L P G, n=14$ and UPG, $n=15$. The study followed the Declaration of Helsinki and was approved by the Universidad de Los Llanos general direction of research Villavicencio-Meta (Meeting Minutes $N^{\circ} 31$, August $25^{\text {th }} 2011$ ).

The inclusion criteria were: minimum participation of $80 \%$ in the intervention programs, no musculoskeletal injuries and/ or bone malformations, being in the first periodized training cycle, as well as presenting a medical certificate for participation in physical activities issued by the Medical Department of the Sports Institute of Meta, Colombia, and written approbation from parents for participation in this study. The characteristics of the groups are presented in Table 1. Groups were similar in all characteristics.

Table 1. Means and standard deviations of age, body mass, body height, and training experience by group (LPG=Linear Periodization Group and $\mathrm{UPG}=$ Undulating Periodization Group).

\begin{tabular}{lccc}
\hline & LPG $(\mathbf{n}=\mathbf{1 4})$ & UPG $(\mathbf{n = 1 5})$ & P \\
\hline Age (years) & $10.33 \pm 1.6$ & $9.84 \pm 0.75$ & 0.60 \\
Body mass (kg) & $30.88 \pm 9.89$ & $33.02 \pm 9.49$ & 0.09 \\
Body height (m) & $1.35 \pm 0.16$ & $1.43 \pm 0.09$ & 0.54 \\
Training experience (years) & $1.6 \pm 0.1$ & $1.5 \pm 0.3$ & 0.08 \\
\hline
\end{tabular}

\section{Procedures}

\section{Training Periodization}

The interventions had a duration of 36 sessions over 16 weeks, distributed as follows: 4 weeks of evaluations separated into initial evaluation (E1) at week 0 , second evaluation (E2) at week 5, third evaluation (E3) at week 10, and fourth evaluation (E4) at week 16. The training program was developed in 3 blocks of 4 weeks ( 12 weeks). Both groups performed 3 non-consecutive sessions per week. Each session lasted 45 minutes: 5 minutes of warm-up and 40 minutes for specific work. The regular skating training was performed equally for both groups for 6 days a week.

For the intervention programs, 6 levels of training were designed, with increasing intensity. Both groups used the same types of exercises, maintaining the same training volume, with different loads. At each level of training the following types of exercises and volume were developed:

- $\quad$ Level 1 bipodal-unidirectional: 12 exercises 2 sets $\times 2$ repetitions, with breaks of 30 and $60 \mathrm{~s}$. At this level, the activities were characterized by rapid jumps with both legs at the same time and with a forward trajectory, 4 exercises were carried out per work session;

- Level 2 unipodal-multidirectional: 8 exercises 1 set $x 3$ repetitions with pauses of $30 \mathrm{~s}$ and $60 \mathrm{~s}$. In this level, the exercises had an emphasis on one-leg execution and with variations in the direction of the exercises, 4 exercises were carried out for each work session;

- $\quad$ Level 3 plyometric technique: 8 exercises 2 sets $\mathrm{x} 3$ repetitions with pauses between $90 \mathrm{~s}$ and 4 minutes. The activities of this level were determined as plyometric due to the use of the specific height and the similarity 
that these exercises have with the technical movements used in speed skating ${ }^{11,17}, 4$ exercises were developed for each session of work;

- Level 4 plyometrics with an elastic band: 8 exercises 2 sets $\mathrm{x} 3$ repetitions with pauses between $90 \mathrm{~s}$ and 4 minutes. Exercises developed at this level had a direct relationship with the previous level, and an external resistance (elastic band) was added to increase the difficulty level, 4 exercises were carried out per work session;

- Level 5 towing exercises: 8 exercises 3 repetitions $\mathrm{x}$ 10 seconds with breaks between $90 \mathrm{~s}$ and 4 minutes. Two conditions of exercises were emphasized in this level: wearing shoes and skates with an implemented sled to pull the equivalent of $10 \%$ of the body mass of each individual ${ }^{18}$. The method of contrast or complex training for the exercises was applied on the skates and consisted of pulling another sportsman of the same anthropometric characteristics. In this kind of exercise, working time was divided into, the first 5 seconds of traction at the maximum speed and the remaining 5 seconds trying to reach maximum acceleration without any resistance. All exercises were carried out in each session of work;

- $\quad$ Level 6 Facilitated Exercises: 6 exercises 3 sets x 5 seconds with pauses between 1 and 3 minutes. At this level, the proposed exercises provided outside help in their execution, such as running downhill or running in favor of the strength of previously elongated elastic cords and an increment in movement frequency of specific techniques ${ }^{19}$. All exercises were carried out for each session of work.

The LP was designed in such a way that the intensity training level increased sequentially and varied every two microcycles. The UP was designed through a mixture of increasing levels in each microcycle, considering that in each mesocycle a level with a difference in intensity greater than 2 was not performed (Figure1).

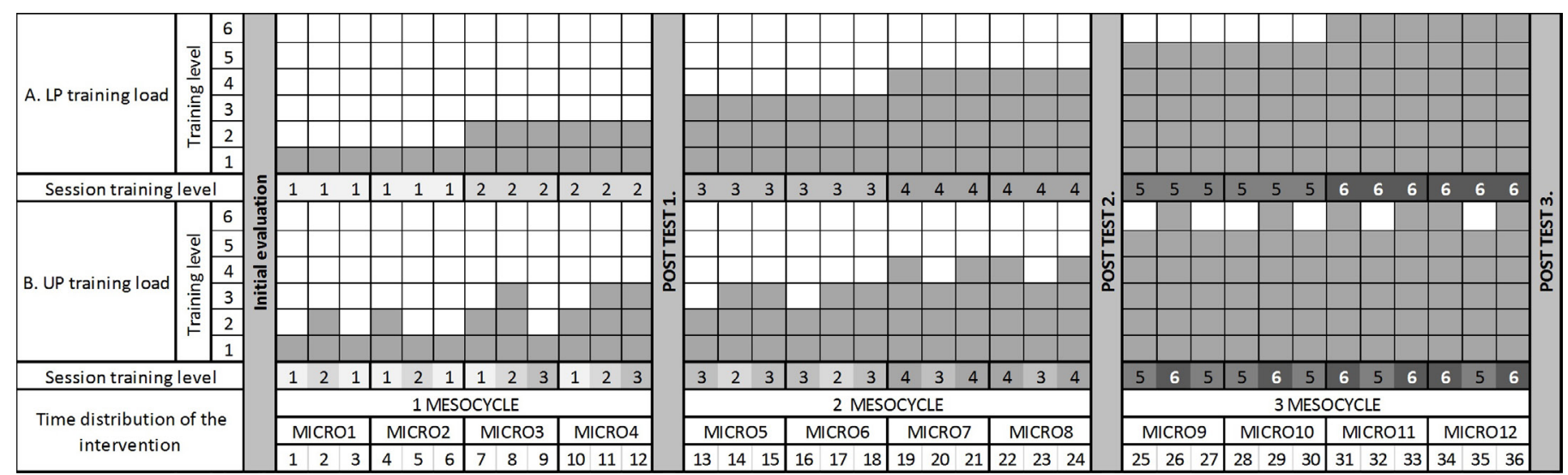

Figure 1. A. Linear Periodization Model (LP); B. Undulating Periodization Model (UP).

\section{Training evaluation}

The velocity and acceleration assessments were performed by filming the frontal and sagittal planes of the participants. Two Sony Handycam (dcr-SX44, $60 \mathrm{~Hz}$ ) cameras were used. The videos of interest were edited and the variables analyzed with Silicon Coach software. Trajectories of the major trochanter of the femur were used to extract the variables of interest.

The measurements took place on a $200 \mathrm{~m}$ skating rink. The specific evaluation spot was in the rink's straight lane. The static start (SS) with voluntary initiation was evaluated at a distance of $10 \mathrm{~m}$. For the dynamic start (DS), the skater was required to maintain $60 \%$ of the maximum speed provided by the trainer during 2 laps of $400 \mathrm{~m}$, with the speed controlled by an experienced skater. In order to measure the acceleration over $20 \mathrm{~m}$, the guide skater left the trajectory of the subject, allowing her to increase speed as fast as possible. Five trials were performed for SS and DS, with a recovery interval between trials of 2 minutes for SS and 4 minutes for DS.

\section{Statistical Analysis}

Data analysis was performed in SPSS software version 21.0. The level of significance was set at $5 \%$ for all analysis. In order to verify the effect of the interventions, a two-way ANOVA (group X moment) with repeated measures for the moment factor was performed for each start type. The Bonferroni post hoc test was used to verify the differences between factors when the interaction was indicated in the analysis. The partial eta squared $\left(\mathrm{p} \eta^{2}\right)$ statistic was used to estimate effect sizes.

\section{Results}

Univariate analysis showed interactions between group and moment for velocity in $10 \mathrm{~m}\left(\mathrm{~F}_{3.81}=7.883 ; \mathrm{p}<0.001 ; \mathrm{p}^{2}=0.226\right)$ and acceleration in $10 \mathrm{~m}$ for $\mathrm{SS}\left(\mathrm{F}_{3,81}=3.96 ; \mathrm{p}=0.011 ; \mathrm{p}^{2}=0.128\right)$ (Figure $2 \mathrm{a}, \mathrm{c}$ ). The post hoc tests showed that, in the $10 \mathrm{~m}$ path, UPG increased velocity and acceleration at the $2 \mathrm{nd}(\mathrm{p}<0.001$, $\mathrm{p}=0.001$, respectively), $3 \mathrm{rd}(\mathrm{p}<0.001, \mathrm{p}<0.001$, respectively), 
and 4th moments ( $\mathrm{p}<0.001, \mathrm{p}<0.001$, respectively) compared to the $1 \mathrm{st}$ moment. In addition, the UPG presented higher acceleration at the $2 \mathrm{nd}$ moment than the $3 \mathrm{rd}(\mathrm{p}=0.022)$ and 4 th moments $(p=0.046)$. The LPG demonstrated increased velocity at the $2 \mathrm{nd}(\mathrm{p}=0.001), 3 \mathrm{rd}(\mathrm{p}<0.001)$, and 4th moments $(\mathrm{p}<0.001)$ compared to the $1 \mathrm{st}$ moment. In addition, the LPG showed higher acceleration at the $3 \mathrm{rd}(\mathrm{p}<0.001)$ and 4 th moments $(\mathrm{p}<0.001)$ in relation to the $1 \mathrm{st}$ moment, and at the 4 th moment compared to the 3rd moment $(p=0.032)$. The UPG showed higher velocity and acceleration at the $2 \mathrm{nd}(\mathrm{p}=0.007, \mathrm{p}=0.045$, respectively), 3rd ( $p=0.01, p=0.006$, respectively), and 4th moments $(p=0.02$, $\mathrm{p}=0.037$, respectively) compared to the LPG. a)

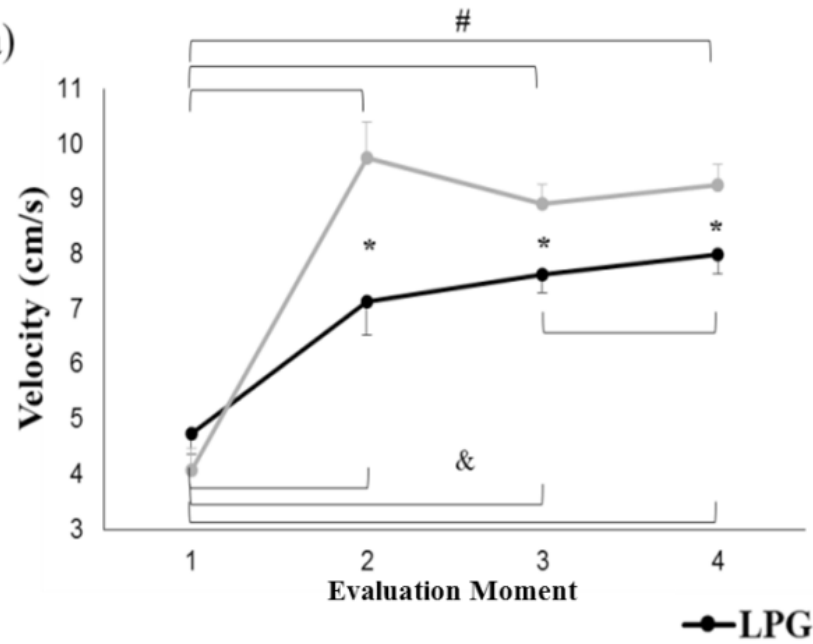

c)

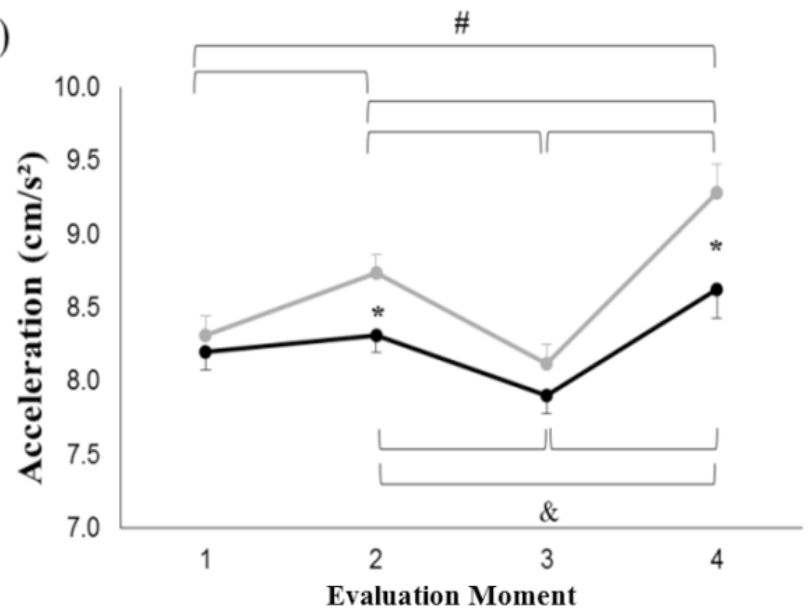

b)
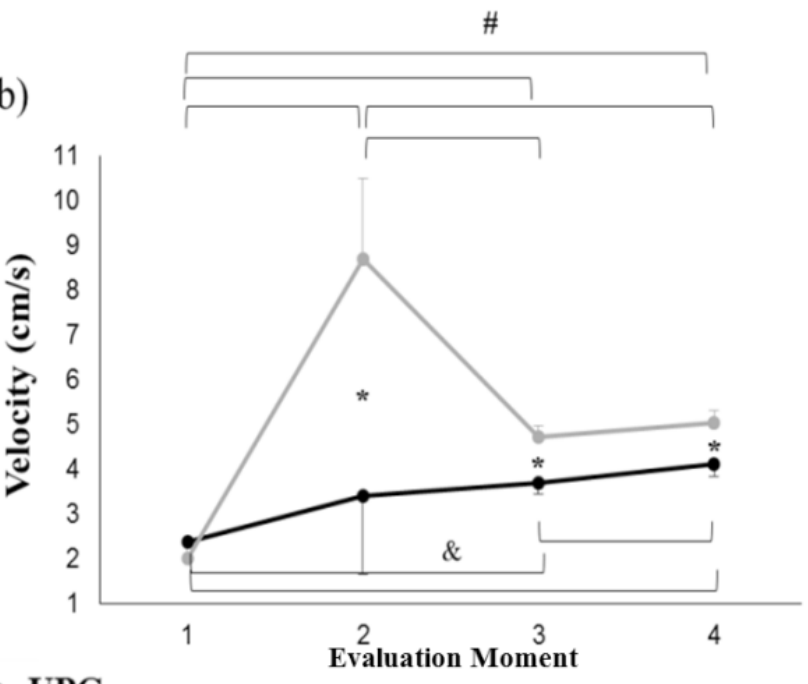

离

$\#$

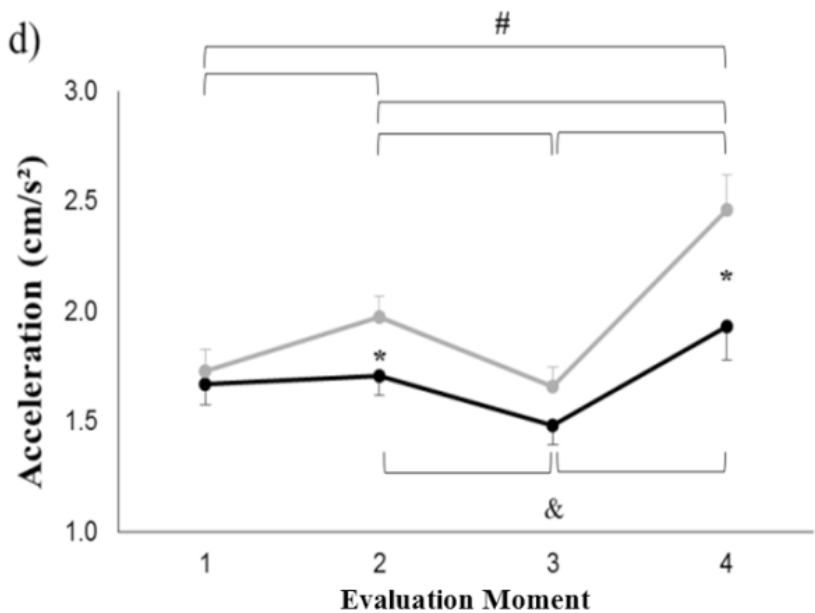

Figure 2. Interaction between group and moment for: a) velocity in $10 \mathrm{~m} \mathrm{SS}$; c) acceleration in $10 \mathrm{~m} \mathrm{SS}$; b) velocity in $20 \mathrm{~m}$ DS; d) acceleration in $20 \mathrm{~m}$ DS

* difference between groups; \# difference between moments for UPG; \& difference between moments for LPG.

With regard to the DS, ANOVA demonstrated an interaction between group and moment for velocity in $20 \mathrm{~m}\left(\mathrm{~F}_{3,81}=2.36\right.$; $\mathrm{p}=0.078 ; \mathrm{p \eta}^{2}=0.08$ - trend $)$ and acceleration in $20 \mathrm{~m}\left(\mathrm{~F}_{3,81}=2.92\right.$; $\mathrm{p}=0.039 ; \mathrm{p}^{2}=0.098$ ) (Figure $\left.2 \mathrm{~b}, \mathrm{~d}\right)$. The post hoc tests showed that the UPG increased velocity and acceleration at the 4 th moment in relation to the $1 \mathrm{st}(\mathrm{p}<0.001, \mathrm{p}<0.001$, respectively), 2nd ( $\mathrm{p}=0.001, \mathrm{p}=0.001$, respectively), and 3rd moments ( $<0.001$, $\mathrm{p}<0.001$, respectively) and at the $2 \mathrm{nd}$ moment showed higher velocity and acceleration than the $1 \mathrm{st}(\mathrm{p}=0.001, \mathrm{p}=0.007$, respectively) and 3 rd moments ( $\mathrm{p}<0.001, \mathrm{p}=0.001$, respectively). The LPG presented higher velocity and acceleration at the 2nd $(p=0.001, p=0.014$, respectively) and 4 th moments $(p<0.001$, $\mathrm{p}=0.001$, respectively) compared to the $3 \mathrm{rd}$ moment. In addition, the LPG demonstrated higher velocity at the 4th moment than the 2 nd moment $(\mathrm{p}=0.049)$. The UPG showed higher velocity and acceleration at the $2 \mathrm{nd}(\mathrm{p}=0.017, \mathrm{p}=0.05$, respectively) and 4 th moments ( $\mathrm{p}=0.028, \mathrm{p}=0.025$, respectively) compared to the $\mathrm{LPG}$.

\section{Discussion}

To our knowledge, this is the first study to investigate strength training periodization models in speed skaters. Thus, the purpose of this study was to analyze the effects of LP and UP 
models in strength training on acceleration performance in skater children. The results showed a positive effect of the implemented intervention, suggesting that UP of training load intensity causes greater gains in speed and acceleration of 10 and 20 meters when compared with the gains generated by LP (Figure 2). These results corroborate with other studies that observed an increase in the yield and development of these variables with high and low-velocity resistance training in sprinter runners ${ }^{20}$, integrative neuromuscular training in children ${ }^{4}$, and a strength training program in children and adolescents ${ }^{3}$. In addition, strengthtraining exercises for young athletes in conditions similar to the mechanics of the sport technique contribute to improving the neuromuscular component intra and intermuscular adaptations ${ }^{21}$ necessary to increase technique performance in the sport.

The major findings of this study show that UP generates the level of stress necessary to provide gains in the analyzed parameters, by manipulating the intensity of training in a population of child athletes. These behavioral improvements have been demonstrated in other studies, with similar training load manipulation in different populations ${ }^{22-26}$. Another new aspect of the training protocol in the present study is the integration of different strength-training methodologies that, although considered safe and efficient, have until now been poorly explored with child populations ${ }^{8,19,27-29}$ and that generated positive effects in the outcome measures. Unlike Kobal and colleagues ${ }^{23}$, who reported no significant differences by combining different methods of resisted and plyometric training in the same session, on muscular strength, jumping plyometrics, speed and agility in elite young soccer players, our results for speed and acceleration under SS and DS conditions in skater children may be generated by the integration and interconnection of the different training stimuli developed in UP in our long duration intervention.

This study revealed that UP is more effective than LP in achieving improvements in speed performance, demonstrating mean increases in speed of $10 \mathrm{~m}(128.5 \%)$ and $20 \mathrm{~m}(59.8 \%)$, respectively, among the four evaluation moments. UPinduced adaptations are mainly attributable to increases in neuromuscular performance due to the specificity of the intervention protocol and low levels of testosterone presented by this population in the maturation phase, which implies a low response in muscular structural adaptations ${ }^{30}$. However, Michailidis et al. ${ }^{3}$ found that the combination of plyometric training and soccer-specific training in prepubertal soccer players significantly increased performance in running speed of $10 \mathrm{~m}(40.6 \%)$ and $20 \mathrm{~m}(12.0 \%)$, which consolidates the idea that combined training generates positive results in speed performance. Interestingly, the specific soccer training control group also demonstrated significant increases in running speed of $10 \mathrm{~m}(14.9 \%)$ suggesting that sport specific training may be sufficient for the development of speed. According to our results, the intensity of the training load under UP is the only component of the periodization that justifies the magnitude of increase presented in the result parameters. As the improvement presented in other studies and in the LPG are within expectations generated by LP interventions, the evidence presented in the UPG data overcomes the impact of the sports training generated by the LP intervention, demonstrating greater increases in acceleration for SS and DS, indicating that UP can justify the observed improvements in the $\mathrm{UPG}^{31}$.

Our results related to percentage gains in acceleration performance for SS and DS are in agreement with the literature ${ }^{7,8,15,17,28,32-34}$, which indicates that strength training focused on acceleration seems to be an efficient and safe way to increase children's acceleration performance. The effects generated by the applied protocols are evidenced by two major components: muscle power ${ }^{35}$ and intra and intermuscular coordination $^{36}$. From these results, it can be inferred that the increases evidenced in the acceleration of the groups are supported by positive adaptations in muscle power of the lower limbs, as has been observed in previous studies ${ }^{36-38}$. The methods linked in the intervention proposal of the present study generate increases in motor performance from positive neuromuscular adaptations that improve muscle power. Thus, the results indicate that UP is more efficient for the improvement in this variable compared to LP, taking into account that the only differentiating factor in the interventions for each group was the training load periodization model.

The results suggest that frequent changes in UP intensity can optimize the effects on neural pathways related to strength development and control of movement, facilitating a positive transfer of strength gains to the specific functionality of the sport and improving the mechanics of movement execution ${ }^{22}$. These benefits in the execution of the technique are associated with improvements in movement control, due to increases in intermuscular coordination ${ }^{39,40}$ and the capacity to generate force, facilitated by better cortical activity ${ }^{12}$, possibly promoted by the stimulating characteristics of UP ${ }^{21}$. On the other hand, it can be inferred that the absence of a significant yield in LPG may be associated with the lack of interconnection of different intensities generated by the isolated application of assisted exercise from another overloaded method. Therefore, LP is not able to promote the best efficiency in the training of acceleration capacity in comparison with the overload stimuli raised by UP, which consider the combination of the specific effects of the exercise methods implemented in each level of training.

This study demonstrated that speed and acceleration variables for 10 and $20 \mathrm{~m}$ improved in pre-pubertal skaters after 16 weeks of strength training composed of multiple jumps, plyometric and towing methods, with a UP design. These results are in agreement with previous reports about significant adaptations after plyometric training of 4 to 6 weeks ${ }^{11,22,29,37}$. In addition, other studies using the sled towing method have demonstrated improvements in acceleration performance after isolated interventions of 6-week duration ${ }^{38,41-43}$. The long duration of our UP intervention makes the combination of training methods possible, joining their isolated effects evidenced in laboratory and field studies and showing greater effectiveness than the application of the methods in isolation. Our results confirm our hypothesis that training with UP generates greater gains in acceleration in skater children than LP.

In conclusion, the results obtained from this study suggest that UP in strength training is more efficient for developing speed and acceleration performance in child skaters compared to LP. 


\section{Pratical Aplications}

The results obtained in the present study indicated that strength training with UP conducted in conjunction with regular inline skate training is safe and feasible for skater children and more efficient than the same training with LP for stimulating acceleration for SS and DS after a 16-week strength training intervention. This finding could help coaches to increase the efficiency of their sports training process, however, they should exercise caution when using this intervention model, by modifying levels 3 and 4, as these levels involve plyometric exercises related to inline skate technique, in order to apply this periodization model in other sports and ensure the specificity training principle. The authors believe that with these modifications, this periodization model could be applied in other sports, and specifically, according to the duration of this study, UP can be used in the specific preparation phase in a longer training process.

\section{References}

1. Behringer M, Vom Heede A, Matthews M, Mester J. Effects of strength training on motor performance skills in children and adolescents: a meta-analysis. Pediatr Exerc Sci. 2011;23(2):186-206.

2. Faigenbaum AD, Myer GD, Farrell A, Radler T, Fabiano M, Kang $\mathrm{J}$, et al. Integrative neuromuscular training and sex-specific fitness performance in 7-year-old children: an exploratory investigation. J Athl Train. 2014;49(2):145-53.

3. Michailidis Y, Fatouros IG, Primpa E, Michailidis C, Avloniti A, Chatzinikolaou A, et al. Plyometrics' trainability in preadolescent soccer athletes. J Strength Cond Res. 2013;27(1):38-49.

4. Faigenbaum AD, Farrell A, Fabiano M, Radler T, Naclerio F, Ratamess NA, et al. Effects of integrative neuromuscular training on fitness performance in children. Pediatr Exerc Sci. 2011;23(4):573-84.

5. Faigenbaum AD, Lloyd RS, Myer GD. Youth resistance training: past practices, new perspectives, and future directions. Pediatr Exerc Sci. 2013;25(4):591-604.

6. Behringer M, Vom Heede A, Yue Z, Mester J. Effects of resistance training in children and adolescents: a meta-analysis. Pediatrics. 2010;126(5):1199-210.

7. Cronin JB, Hansen KT. Strength and power predictors of sports speed. J Strength Cond Res. 2005;19(2):349-57.

8. Lloyd RS, Faigenbaum AD, Stone MH, Oliver JL, Jeffreys I, Moody JA, et al. Position statement on youth resistance training: the 2014 International Consensus. Br J Sports Med. 2014;48(7):498-505.

9. Apel JM, Lacey RM, Kell RT. A comparison of traditional and weekly undulating periodized strength training programs with total volume and intensity equated. J Strength Cond Res. 2011;25(3):694-703.

10. Faigenbaum AD. Strength training for children and adolescents. Clin Sports Med. 2000;19(4):593-619.

11. Faigenbaum AD, McFarland JE, Keiper FB, Tevlin W, Ratamess NA, Kang J, et al. Effects of a short-term plyometric and resistance training program on fitness performance in boys age 12 to 15 years. J Sports Sci Med. 2007;6(4):519-25.
12. Jensen J, Marstrand P, Nielsen J. Motor skill training and strength training are associated with different plastic changes in the central nervous system. J Appl Physiol. 2005;99(4):1558-68.

13. Issurin V. New horizons for the methodology and physiology of training periodization. Sports Med. 2010;40(3):189-206.

14. Contreras D, Rojas D, Palomino O. Effects of two periodizations form (linear and double undulated) in strength training on the kinematics of the voluntary static start in pre-pubescent skaters. (Efectos de dos formas de periodizar la carga (lineal y doble ondulada) en el entrenamiento de la fuerza, sobre la cinemática de la salida estática voluntaria en patinadores de carreras pre púberes). Revista Actividad Física y Desarrollo Humano. 2012;4(1):88-96.

15. Ullrich B, Pelzer T, Oliveira S, Pfeiffer M. Neuromuscular responses to short-term resistance training with traditional and daily undulating periodization in adolescent elite judoka. J Strength Cond Res. 2016;30(8):2083-99.

16. Harries S, Lubans D, Callister R. Systematic review and meta-analysis of linear and undulating periodized resistance training programs on muscular strength. J Strength Cond Res. 2015;29(4):1113-25.

17. Sáez-Sáez de Villarreal E, Requena B, Newton R. Does plyometric training improve strength performance? A meta-analysis. J Sci Med Sport. 2010;13(5):513-22.

18. Alcaraz PE, Palao JM, Elvira JLL. Determining the optimal load for resisted sprint training with sled towing. J Strength Cond Res. 2009;23(2):480-5.

19. Alcaraz, PE. Sprint training with resitive methods (El entrenamiento del sprint con métodos resistidos). Cultura, Ciencia y Deporte. 2010;5(15):19-26.

20. Blazevich A, Jenkins D. Effect of the movement speed of resistance training exercises on sprint and strength performance in concurrently training elite junior sprinters. J Sport Sci. 2002;20(12):981-90.

21. Carroll T, Riek S, Carson R. Neural adaptations to resistance training. Sports Med. 2001;31(12):829-40.

22. Herrero J, Izquierdo M, Maffiuletti N, Garcia-Lopez J. Electromyostimulation and plyometric training effects on jumping and sprint time. Int J Sports Med. 2006;27(07):533-9.

23. Kobal R, Loturco I, Barroso R, Gil S, Cuniyochi R, Ugrinowitsch $\mathrm{C}$, et al. Effects of different combinations of strength, power, and plyometric training on the physical performance of elite young soccer players. J Strength Cond Res. 2017;31(6):1468-76.

24. Michaleff ZA, Kamper SJ. Effects of resistance training in children and adolescents: a meta-analysis. Br J Sports Med. 2011;45(9):755.

25. Ramírez-Campillo R, Meylan C, Álvarez-Lepín C, HenriquezOlguín C, Martinez C, Andrade D, et al. The effects of interday rest on adaptation to 6 weeks of plyometric training in young soccer players. J Strength Cond Res. 2015;29(4):972-9.

26. Spinks C, Murphy A, Spinks W, Lockie R. The effects of resisted sprint training on acceleration performance and kinematics in soccer, rugby union, and Australian football players. J Strength Cond Res. 2007;21(1):77-85.

27. Alcaraz P, Elvira J, Palao J. Caracteristics and effects of resistive methods in sprint (Características y efectos de los métodos resistidos en el sprint). Cultura, Ciencia y Deporte. 2009;4(12):179-87.

28. Alcaraz P, Palao J, Elvira J, Linthorne N. Effects of three types of resisted sprint training devices on the kinematics of sprinting at maximum velocity. J Strength Cond Res. 2008;22(3):890-7. 
29. Meylan C, Malatesta D. Effects of in-season plyometric training within soccer practice on explosive actions of young players. J Strength Cond Res. 2009;23(9):2605-13.

30. Luebbers P, Potteiger J, Hulver M, Thyfault J, Carper M, Lockwood R. Effects of plyometric training and recovery on vertical jump performance and anaerobic power. J Strength Cond Res. 2003;17(4):704-9.

31. Fleck S. Non-linear periodization for general fitness \& athletes. J Hum Kinet. 2011;29(Special Issue):41-5.

32. Lockie RG, Murphy AJ, Schultz AB, Knight TJ, Janse de Jonge XA. The effects of different speed training protocols on sprint acceleration kinematics and muscle strength and power in field sport athletes. J Strength Cond Res. 2012;26(6):1539-50.

33. Markovic G, Mikulic P. Neuro-musculoskeletal and performance adaptations to lower-extremity plyometric training. Sports Med. 2010;40(10):859-95.

34. Monteiro A, Aoki M, Evangelista A, Alveno D, Monteiro G, da Cruz Piçarro I, et al. Nonlinear periodization maximizes strength gains in split resistance training routines. J Strength Cond Res. 2009;23(4):1321-6.

35. Johnson M, Buckley J. Muscle power patterns in the mid-acceleration phase of sprinting. J Sport Sci. 2001;19(4):263-72.

36. Behm D, Young J, Whitten J, Reid J, Quigley P, Low J, et al. Effectiveness of traditional strength vs. power training on muscle strength, power and speed with youth: a systematic review and meta-analysis. Front Physiol. 2017;8:423-60.

37. Diallo O, Dore E, Duche P, Van Praagh E. Effects of plyometric training followed by a reduced training programme on physical performance in prepubescent soccer players. J Sports Med Phys Fit. 2001;41(3):342-8.

38. Petrakos G, Morin J-B, Egan B. Resisted sled sprint training to improve sprint performance: a systematic review. Sports Med. 2016;46(3):381-400.

39. Ozmun J, Mikesky A, Surburg P. Neuromuscular adaptations following prepubescent strength training. Med Sci Sport Exercise. 1994;26(4):510-4.

40. Myer G, Ford K, Palumbo J, Hewett T. Neuromuscular training improves performance and lower-extremity biomechanics in female athletes. J Strength Cond Res. 2005;19(1):51-60.
41. Cronin J, Hansen K, Kawamori N, Mcnair P. Effects of weighted vests and sled towing on sprint kinematics. Sports Biomech. 2008;7(2):160-72.

42. Lockie RG, Murphy AJ, Spinks CD. Effects of resisted sled towing on sprint kinematics in field-sport athletes. J Strength Cond Res. 2003;17(4):760-7.

43. Clark K, Stearne D, Walts C, Miller A. The longitudinal effects of resisted sprint training using weighted sleds vs. weighted vests. J Strength Cond Res. 2010;24(12):3287-95.

\section{Acknowledgments}

The authors wish to thank: all the subjects and their parents for their participation and commitment to the study; Rubiel Barrera, Neal Salamanca, Stephania Moreno, Eduardo Sarmiento, and all the student members of the Human Movement Sciences Research Group; and Los Llanos University's Department of Physical Education and Faculty of Education. This study was supported by the Los Llanos University Research Department.

\section{Corresponding author}

Diego A. R. Jaimes

Address: Avenue 24 A,1515 CEP: 13506900, Rio Claro São Paulo State, Brazil

Email: darjaimes02@hotmail.com

Manuscript received on May 17, 2018

Manuscript accepted on October 1, 2018

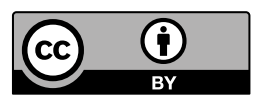

Motriz. The Journal of Physical Education. UNESP. Rio Claro, SP, Brazil - eISSN: 1980-6574 - under a license Creative Commons - Version 4.0 